\title{
Design and research of hybrid cloud desktop scheme in colleges and universities
}

\author{
Nian Xiong ${ }^{1}$, Shan Zhou ${ }^{1}$, Zujian $\mathrm{Wu}^{2}$, and Zhen Zhang ${ }^{1, *}$ \\ ${ }^{1}$ College of Information Science and Technology, Jinan University, 601 West Huangpu Ave, \\ Guangzhou, China \\ ${ }^{2}$ College of Birmingham Joint Institute, Jinan University, 601 West Huangpu Ave, Guangzhou, China
}

Keywords: Cloud desktop, VDI, VOI.

\begin{abstract}
This paper analyzes and compares the four mainstream cloud desktop technologies: RDS, VDI, IDV and VOI. According to the practical application in colleges and universities, a hybrid cloud desktop technology scheme is designed and proposed, and the specific implementation method of the integration of VDI and VOI is studied. In view of the particularity and requirements of experimental courses and application scenarios, an application model based on this technical scheme is designed, which is suitable for all the common scenes of computer experimental teaching, realizes the deep integration of cloud desktop and teaching activities, and also provides reference for the construction and application of cloud desktop technology in colleges and universities.
\end{abstract}

\section{Introduction}

Campus cloud construction is an important development direction of university information construction, and cloud desktop technology is the most typical and important application of cloud computing. According to International Data Company(IDC)'s forecast, the cloud desktop market will continue to grow rapidly in the next five years, with a compound annual growth rate(CAGR) of $44.3 \%$ [1]. At present, the application of computer technology in various disciplines is becoming more and more extensive. It is a general trend for colleges and universities to build cloud desktop system based on virtualization technology. As an important part of the campus cloud, the computer public laboratory of our university is open to the whole school, with 10 computer rooms and nearly 1000 computers, including various types of laboratories such as traditional computer and cloud computing. It is a necessary process of laboratory construction and experimental technology development to integrate computer resources and build a scientific and unified management of virtual teaching environment. Based on the practical application in colleges and universities, this paper designs and proposes a hybrid cloud desktop technology scheme, and studies the practical application model based on the scheme.

\footnotetext{
* Corresponding author: nancy0122@163.com
} 


\section{Cloud desktop technology}

Cloud desktop is a cloud computing technology solution that uses virtualization technology to push virtual desktop in remote server to different types of clients through network. [1],[2].

\subsection{Technical architectures}

According to the virtualization architecture, the mainstream cloud desktop can be divided into four categories: RDS (Remote Desktop Service), VDI (Virtual Desktop Infrastructure), IDV (Intelligent Desktop Virtualization) and VOI(virtual OS Infrastructure)[3]. RDS is based on a multi-user operating system. It configures servers according to the number of users, creates users in batches, and distributes them to each client using cloud desktop transport protocol. All users share a set of operating system and software, which has certain user restrictions and limitations. VDI installs and runs hypervisor on the physical server through virtualization technology, deploys the resource pool of the server into multiple virtual machines on demand, and transmits the graphical interface of virtual machine to cloud terminal through network protocol. IDV is to virtualize several different operating systems on the server side. According to the requirements, the virtual machine on the server is loaded to the client for local use, and the data of each client is synchronized to the server by using image technology. VOI is the centralized deployment of desktop operating system and application software on the server side, and the operating system and application software are distributed to the client by virtualization container technology on demand, and the virtualization operating system works completely in the local terminal.

\subsection{Features and comparison}

VDI has high security performance, supports multi terminal access, can realize mobile teaching and mobile office, but it has high requirements on server performance, network connectivity and network quality, does not support offline use, and peripheral compatibility is not high. The experimental environment of RDS architecture has low deployment flexibility and limited number of concurrent users, so it is only suitable for a single laboratory. IDV supports offline, with good user experience, but it is highly dependent on the terminal and does not support mobile access to the desktop. Once the terminal fails, the operation and maintenance are difficult. Compared with IDV, VOI saves the virtualization layer, so there is no loss of performance, strong graphics processing ability and the best user experience. VOI is not highly dependent on server performance and stability and network, and has strong compatibility with peripherals. It supports offline operation, but does not support multi terminal access, and has high coupling degree with the hardware environment of the terminal.

Table 1. The Main Features of the Four Architectures.

\begin{tabular}{|c|c|c|c|c|}
\hline Features & RDS & VDI & IDV & VOI \\
\hline $\begin{array}{l}\text { Computing } \\
\text { Form }\end{array}$ & \multicolumn{2}{|c|}{ Server Computing } & \multicolumn{2}{|c|}{ Local Computing } \\
\hline $\begin{array}{l}\text { Maintenance } \\
\text { Management }\end{array}$ & \multicolumn{2}{|c|}{ Server Maintenance } & \multicolumn{2}{|c|}{ Server and Terminal Maintained } \\
\hline $\begin{array}{c}\text { Network } \\
\text { Dependence }\end{array}$ & \multicolumn{2}{|c|}{$\begin{array}{l}\text { High speed network, dependent } \\
\text { network }\end{array}$} & $\begin{array}{c}\text { High speed } \\
\text { network, } \\
\text { support offline }\end{array}$ & $\begin{array}{l}\text { Medium speed } \\
\text { network, } \\
\text { support offline }\end{array}$ \\
\hline $\begin{array}{l}\text { Image } \\
\text { Performance }\end{array}$ & \multicolumn{3}{|c|}{ General, no 3D support } & $\begin{array}{l}\text { Excellent, 3D } \\
\text { support }\end{array}$ \\
\hline Terminal Access & $\begin{array}{l}\text { limited mobile } \\
\text { access Support }\end{array}$ & $\begin{array}{l}\text { mobile access } \\
\text { Support }\end{array}$ & \multicolumn{2}{|c|}{ No Mobile access support } \\
\hline
\end{tabular}




\section{Scheme design of hybrid cloud desktop}

\subsection{Requirement analysis}

At present, the teaching range of computer experimental courses in colleges and universities is more and more extensive, almost all students have to learn different types of computer experimental courses. Take our university as an example, $30 \%$ of the undergraduates need to complete more than 10 computer experiment courses in four years, $25 \%$ of the undergraduates need to complete 5-10 computer experiment courses, and $45 \%$ of the undergraduates need to complete 1-4 computer experiment courses. There are many kinds of computer equipment in university laboratories. The demand for cloud desktop is higher for thin terminals and computers with poor performance. In the new situation, students' learning habits have changed. After leaving the experimental class, they hope to carry out the unfinished practice in class and even more innovative practice activities. On the other hand, in recent years, there are more and more courses related to 3D modeling and simulation .In the teaching activities with high performance requirements, VDI has limitations, and VOI can better adapt to it. With the development of teaching and technology, a single technology architecture can't meet the actual needs. The construction of hybrid cloud desktop platform is a better solution.

\subsection{System design}

As shown in Figure 1, the hybrid cloud desktop system model is divided into five layers: infrastructure layer, platform layer, desktop layer, terminal layer and user layer.

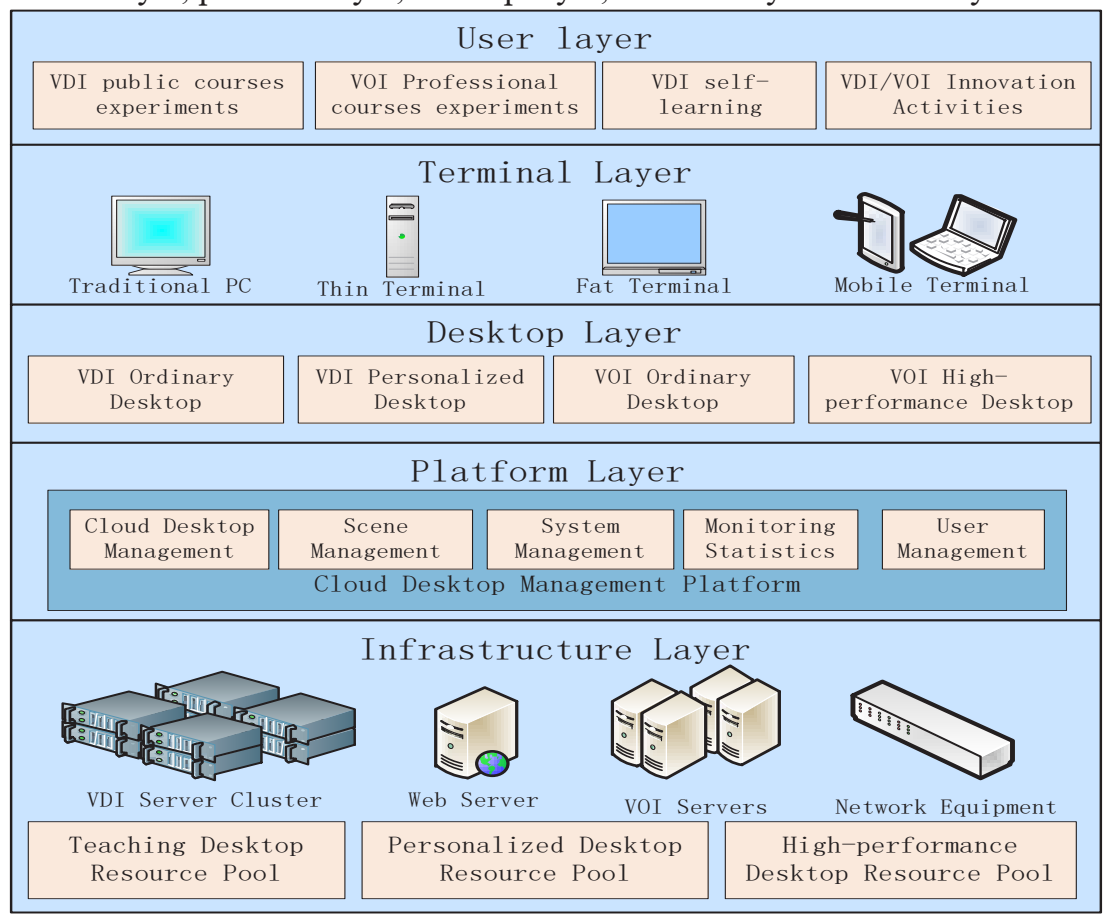

Fig. 1. Hybrid cloud desktop system model.

(1) Infrastructure layer. In the aspect of VDI server deployment, the operating system and 
VDI server-side software are installed, the server-side cluster management environment is established, the basic template of virtual desktop is created, and the special software of different courses is installed on the basic template, and the course template is established[5]. In terms of VOI server deployment, each laboratory is configured with one server. Install system and software, establish system template for multiple operating systems of different laboratory equipment, and configure special software. The server is responsible for saving and managing the template image, and issuing updates to each terminal. In addition, the cloud desktop application publishing website is deployed in an independent server to realize the cloud desktop application and publishing function, backup and store the system image of VDI and VOI, realize the remote backup of system image, and improve the security.

(2) Platform layer. The cloud server connects VDI and VOI subnets through the cloud desktop management platform, and integrates cloud desktop management, scene management, system building, monitoring statistics and user management functions. According to the workload of physical server and virtual desktop, the resource scheduling strategy is uniformly planned. According to the actual needs of different types of teaching desktop resource pool and personalized desktop resource pool, the energy-saving mode scheduling of load balancing is realized to maximize the utilization of system resources.

(3) Realize the release of VDI teaching desktop, VOI teaching desktop, VDI personalized desktop and VOI personalized desktop. For thin clients or computers with poor performance, VDI deployment is the main way to meet the needs of common software operation and programming experiment teaching, and undertake most of the daily experimental teaching work. For desktop computers with good performance, VOI deployment mode is mainly used to meet the experimental teaching of professional courses such as $3 \mathrm{D}$ modeling, artificial intelligence and virtual simulation, and high-performance personal desktop can also be provided to support innovation activities.

(4) Terminal layer. Connect old PC, thin terminal, high performance desktop computer, personal PC, mobile pad and other mobile terminal devices in traditional laboratory. For old PC, VDI client can be deployed on top of it; for thin terminal and high-performance desktop computer, VDI client and VOI client are deployed. According to teaching requirements, they are connected with corresponding server to allocate appropriate terminal system or link course desktop. For personal laptop and other terminals, VDI client is deployed on demand to access personal desktop in the form of account password. If necessary, each student can apply for access to multiple personal desktops.

(5) User layer. It mainly faces common scenarios such as VDI public courses, VOI professional courses, VDI autonomous learning and VDI/VOI innovation activities. Through the platform layer, the idle cloud desktop resources can be released to the maximum extent, and the cloud resources can be accessed at anytime and anywhere through PC client, web, mobile client, etc., so as to conduct personalized learning research.

The hybrid cloud desktop technology scheme breaks the technical boundary between VDI and VOI, and has the mobile characteristics of VDI desktop and the high performance of VOI desktop. In view of the diversified scene requirements, network capabilities, hardware resource environment and other factors of the computer laboratory, through unified management and on-demand distribution, it can effectively improve the teaching energy efficiency, user experience and centralized management ability of computer laboratory.

\section{Application of hybrid cloud desktop}

There are a large number of laboratories in colleges and universities, and there are a large number of laboratories for teaching. Therefore, with different professional needs and computing environment, it is necessary to design different types of cloud desktop 
applications according to the course requirements and application scenarios. The application model of hybrid cloud desktop technology scheme is shown in Figure 2.

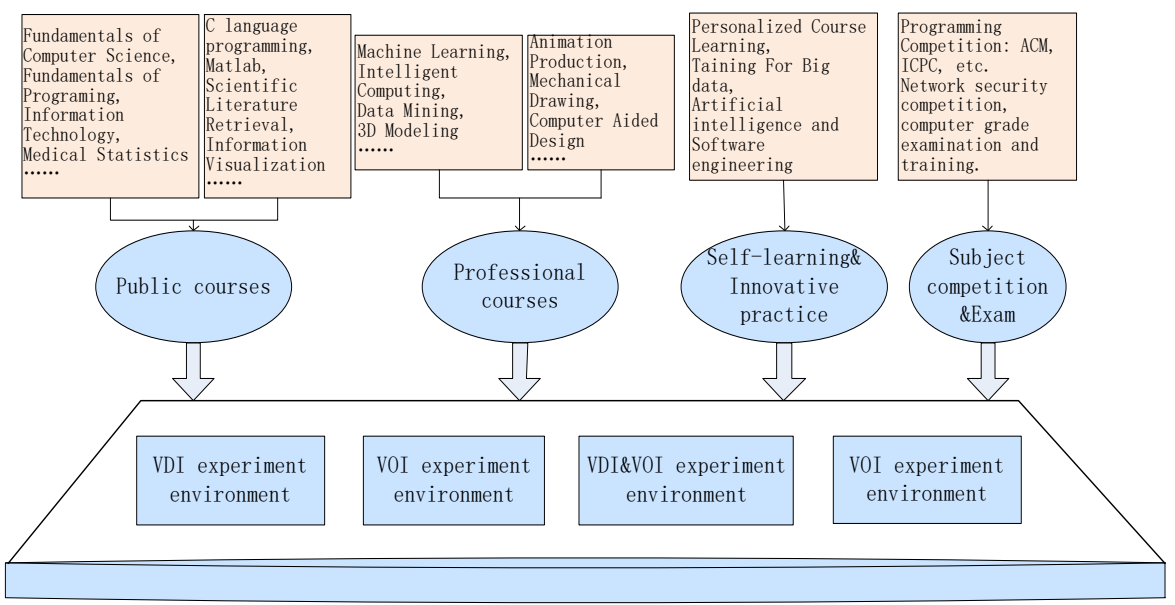

Fig. 2. Application Model.

VDI cloud desktop mode is mostly used in computer public basic courses. VDI can also build a personalized experimental environment to support students' autonomous learning and innovative practice activities. Some professional experimental courses have high requirements on computing performance, which are implemented by VOI cloud desktop mode, such as some courses of intelligent science and technology specialty and design specialty. These courses can achieve the best learning experience through the VOI experimental environment. VOI can also support activities such as high-performance innovation practices and discipline competitions. Build a leading innovative practice learning module on the cloud platform to support professional experimental training and innovative learning activities. At the same time, the construction of a national large-scale computer competition and examination scene environment, forming a high-quality learning closedloop, to achieve the cultivation of students' practical ability and innovation and entrepreneurship literacy.

From the practical application effect of cloud desktop in our school, the hybrid technology scheme can fully cover the needs of experimental courses, and can timely support students' innovation activities. At the same time, maximize the release of idle resources, improve resource utilization and laboratory management efficiency.

\section{Conclusion}

In the information construction of colleges and universities, cloud desktop technology is an important development direction of laboratory construction and experimental technology[6][7]. The education field, especially the universities, should design appropriate technical scheme according to their own construction and management needs. The hybrid cloud desktop technology scheme and application model proposed in this paper realizes the deep integration of cloud desktop and teaching activities, and provides a reference for the development of cloud desktop experimental technology in colleges and universities.

This work is supported in part by the following funding: National Natural Science Foundation (NSF) of China under Grant (No. 61572232), Natural Science Foundation of Guangdong under Grant (No. 2020A1515010619), Laboratory Research Project of Jinan University (No. 2020030) and Teaching Reform Research Project of Jinan University (No. JG2020145). Zhen Zhang is the corresponding author. 


\section{References}

1. Azzedin F,Yahya S,Mahmood S. Performance evaluation of VDI-Based private cloud technology for education and research. International Journal of Computer Science and Network Security. 2019,vol 19.pp 231-237.

2. Azzedin F,Yahya S,Ahmed A.Utilizing cloud computing services in reaching and research. International Journal of Computer Science and Network Security.2019,vol 19.pp 223-230.

3. H. MA,R. MT,H. N,N. MA,S. M. Implementation of VDI based computer laboratory in university education system to save energy cost, and Adapt Technology Upgradation. International Journal of Computer Science and Network Security.2020, vol 20.pp 130137.

4. Chang CH, Yang CT, Lee JY, Lai CL, Kuo CC. On construction and performance evaluation of a virtual desktop infrastructure with GPU accelerated. IEEE Access.2020,vol 8.pp170162-170173.

5. WangH,XueYJ.Research on the application of hybrid cloud desktop design method.M MME 2016 Proc. pp 23-27.

6. Y. Jin, J. Zhu, H.i Bai, H.g Chen, N. Sun. Design of Virtual Cloud Desktop System Based on OpenStack. FITAT 2019 Proc. pp 393-401.

7. Pan Huifang,Yuan Yi,Song Wenlong,An Zhou. The design and implementation of secure cloud desktop system.ICSINC 2019 Proc.pp 212-218. 